\title{
Integrated control performance of drive-by-wire independent drive electric vehicle
}

\author{
Ling $\mathrm{Yu}^{1, *}$ and Sunan $\mathrm{Yuan}^{2}$ \\ ${ }^{1}$ School of Mechanical Engineering, Henan Polytechnic Institute, Nanyang, Henan 473009, PR China \\ ${ }^{2}$ School of Electromechanical Automation, Henan Polytechnic Institute, Nanyang, Henan 473009, PR China
}

Received: 10 October 2019 / Accepted: 9 November 2019

\begin{abstract}
In order to improve the stability and safety of vehicles, it is necessary to control them. In this study, the integrated control method of drive-by-wire independent drive electric vehicle was studied. Firstly, the reference model of electric vehicle was established. Then, an integrated control method of acceleration slip regulation (ARS) and direct yaw moment control (DYC) was designed for controlling the nonlinearity of tyre, and the simulation experiment was carried out under the environment of MATLAB/SIMULINK. The results showed that the vehicle lost its stability when it was uncontrolled; under the control of a single DYC controller, $r$ and $\beta$ values got some control, but the vehicle stability was still low; under the integrated control of ARS $+\mathrm{DYC}$, the vehicle stability was significantly improved; under the integrated control method, the overshoot, regulation time and steady-state error of the system were all small. Under the simulation of extreme conditions, the integrated control method also showed excellent performance, which suggested the method was reliable. The experimental results suggests the effectiveness of the integrated control method, which makes some contributions to the further research of the integrated control of electric vehicles.
\end{abstract}

Keywords: Independent drive electric vehicle / linear control / direct yaw moment control / integrated control

\section{Introduction}

Electric vehicles are vehicles driven by electricity [1], including four-wheel independent drive (4WID), fourwheel independent steering (4WIS), four-wheel independent braking (4WIB), etc. The whole vehicle system adopts wire control technology, which can reduce the load of drivers. In order to further improve the stability and safety of electric vehicles, vehicle stability control has been widely studied. At present, there are two main directions: one is to control the wheel angle, including Active Front Steering (AFS) [2], Active Rear-Wheel Steering (ARS), etc., and the other is to improve the tire adhesion to the ground or to correct the vehicle deficiencies under extreme conditions, including Acceleration Slip Regulation (ASR), Direct Yaw Moment Control (DYC), etc [3]. But these control methods all have some shortcomings. In order to achieve higher performance, different control methods are integrated. Integrated control of vehicles becomes a new choice of electric vehicle control. Integrated control can achieve the optimization of vehicle performance very well, which has attracted extensive attention of researchers. Wu et al. [4] proposed an integrated control strategy combining AFS

\footnotetext{
* Corresponding author: yllingyu@yeah.net
}

and DYC, and developed a two-lane transformation (DLC) simulation, and found through simulation that the method had high control performance. Zhao et al. [5] established a 14-DOF vehicle model, then studied the coordinated control among active suspension system (ASS), AFS and DYC and found that the method could effectively improve the lateral and longitudinal dynamics of vehicles. Shen et al. [6] combined four-wheel steering (4WS) with DYC to ensure vehicle stability by generating appropriate 4WS angle and correcting yaw moment based on sliding mode control and verified the effectiveness of the method through experiments. In this study, an integrated control method combining ARS with DYC was designed for 4WID to improve the control effect when the tyre was in the non-linear state. The simulation results proved the control performance of the method, which is beneficial to improve vehicle stability and safety. This work provides some theoretical bases for improving the control performance of electric vehicles.

\section{4WID model establishment}

4WID electric vehicle [7] was taken as the research subject in this study. The vehicle adopts full drive-by-wire technology, including drive-by-wire driving, steering and 
braking, which can effectively reduce the driver's burden and realize intelligent response of vehicles. It can realize motion modes such as front-wheel steering, rear-wheel steering, oblique motion and crab motion, suggesting excellent performance. Before designing the integrated control method, the reference model of 4WID needs to be designed. In this study, a linear two-degree-of-freedom model was used, and the differential equations are:

$$
m v(\dot{\beta}+r)=-\left(c_{f}+c_{r}\right) \beta-\frac{c_{f} a-c_{r} b}{v} r+c_{f} \delta_{f}+c_{r} \delta r
$$

$$
I_{z} \dot{r}=\left(c_{r} b-c_{f} a\right) \beta-\frac{c_{f} a^{2}+c_{r} b^{2}}{v}+c_{f} a \delta_{f}-c_{r} b \delta_{r},
$$

where:

$m$ : total weight of vehicle, $v$ : centroid speed of vehicle, $\beta$ : sideslip angle of vehicle centroid, $r$ : angular velocity of vehicle, $c_{f}$ : cornering stiffness of front axle, $c_{r}$ : lateral stiffness of back axle, $a$ : distance from centroid to the front axle, $b$ : distance from centroid to the rear axis, $\delta_{f}$ : front wheel steering angle, $\delta_{r}$ : rear wheel steering angle, $I_{z}$ : yaw rotational inertia of vehicle.

According to state quantity $X=[\beta r]^{T}$ and input vector $u=\left[\begin{array}{ll}\delta_{f} & \delta_{r}\end{array}\right]^{T}$, the above equation is converted to state equation, then

$$
\dot{X}=A X+B u
$$

where

$$
A=\left[\begin{array}{cc}
-\frac{c_{f}+c_{r}}{m v} & -1-\frac{c_{f} a-c_{r} b}{m v_{2}} \\
-\frac{c_{f} a-c_{r} b}{I_{z}} & -\frac{c_{f} a^{2}+c_{r} b^{2}}{I_{z} v}
\end{array}\right]
$$

and

$$
B=\left[\begin{array}{rr}
\frac{c_{f}}{m v} & \frac{c_{r}}{m v} \\
\frac{c_{f} a}{I_{z}} & -\frac{c_{r} b}{I_{z}}
\end{array}\right] .
$$

Vehicle reference model is to design expected yaw velocity $r_{d}$ and expected centroid sideslip angle $\beta_{d}$. The steady state response of vehicle yaw angular speed $r$ to front wheel steering angle $\delta_{f}$ can be expressed as:

$$
\begin{gathered}
r=\frac{v}{l+K_{v} v^{2}} \frac{\theta_{s w}}{n_{s w}}=\frac{v}{l+K_{v} v^{2}} \delta_{f}, \\
K_{v}=\frac{m}{l}\left(\frac{b}{c_{f}}-\frac{a}{c_{r}}\right),
\end{gathered}
$$

where $l$ stands for front and rear axle distance, $K_{v}$ stands for vehicle understeering gradient, $\theta_{s w}$ stands for steering angle of steering wheel, and $n_{s w}$ stands for transmission ratio of steering wheel to front wheel.
The maximum lateral acceleration of vehicle will be limited by the ground adhesion coefficient: $a_{y}=\dot{v}_{y}+v_{x} r$, $\left|a_{y} \leq \mu \mathrm{g}\right|$; therefore, the peak expected yaw velocity needs to be limited: $\left|r_{d}\right| \leq\left|r_{\max }\right| \leq 0.85 \frac{u g}{v} \cdot r_{d}$ can be expressed as:

$$
r_{d}=\min \left\{\left|\frac{v}{l+K_{v} v^{2}} \delta_{f}\right|,\left|0.85 \frac{\mu g}{v}\right|\right\} \operatorname{sgn}\left(\delta_{f}\right) \frac{1}{1+\tau_{r} s},
$$

where $a_{y}$ stands for lateral acceleration, $\mu$ stands for nominal ground adhesion coefficient, $g$ stands for gravity acceleration, $\tau_{r}$ stands for first-order delay time, between 0.1 and $0.25 \mathrm{~s}$, and sgn stands for symbolic function.

In order to ensure the high-speed stability of vehicle, suppose $\beta_{d}=0$.

In the reference model, the input is $\theta_{s w}$ or $\delta_{f}$, and the outputs are $r_{d}$ and $\beta_{d}$.

\section{Integrated control method}

\subsection{ARS controller}

ARS means to control the active steering of the rear wheel of a vehicle [8] to reduce the cornering angle and improve vehicle stability, but when the tire is non-linear, its control effect will be weakened. ARS controller is designed with linear sliding mode controller. Its sliding mode surface can be expressed as:

$$
S_{1}=\dot{e}+c_{0} e+c_{1} \int e d t
$$

where $\dot{e}$ stands for the tracking error, $\dot{e}=r-r_{d}$, and $c_{0}$ and $c_{1}$ are undetermined coefficients. Then

$$
\dot{S}_{1}=\dot{r}-\dot{r}_{d}+c_{0} \dot{e}+c_{1} e .
$$

Suppose $\dot{S}_{1}=-K \operatorname{sgn}\left(S_{1}\right)$ and $K$ as the parameter of controller, then:

$$
\begin{aligned}
\delta_{r}= & \frac{I_{z}}{c_{r} l_{r}}\left[\frac{c_{r} l_{r}-c_{f} l_{f}}{I_{z}} \beta-\frac{c_{f} l_{f}^{2}+c_{r} l_{r}^{2}}{I_{z} v} r+\frac{c_{f} l_{f}}{I_{z} \delta_{f}}\right. \\
& \left.-\dot{r}_{d}+c_{0} \dot{e}+c_{1} e+K \operatorname{sgn}\left(S_{1}\right)\right] .
\end{aligned}
$$

To reduce chattering, $\operatorname{sgn}\left(S_{1}\right)$ is replaced by saturation function sat $\left(S_{1}\right)$, then:

$$
\begin{aligned}
\delta_{r}= & \frac{I_{z}}{c_{r} l_{r}}\left[\frac{c_{r} l_{r}-c_{f} l_{f}}{I_{z}} \beta-\frac{c_{f} l_{f}^{2}+c_{r} l_{r}^{2}}{I_{z} v} r+\frac{c_{f} l_{f}}{I_{z} \delta_{f}}\right. \\
& \left.-\dot{r}_{d}+c_{0} \dot{e}+c_{1} e+K \operatorname{sat}\left(S_{1}\right)\right],
\end{aligned}
$$

where $l_{r}$ stands for rear axle distance and $l_{f}$ stands for front axle distance.

According to the above equation, the inputs of ARS controller are $r, \beta, \delta_{f}$ and $r_{d}$, and the output is $\delta_{r}$. 


\subsection{DYC controller}

DYC refers to the control of wheel braking when tyres are non-linear, in details, generating torque to change yaw angular speed. It can improve vehicle yaw stability $[9,10]$ and prevent vehicle from losing control in the non-linear area, but DYC will force to reduce vehicle speed, which is not applicable in some cases where deceleration is not allowed.

When $\beta$ is small, the motion state of vehicle is determined by $r$. Suppose $e=r-r_{d}$. Sliding mode surface is expressed as:

$$
\begin{aligned}
S_{2}=\dot{e}+e= & \left(\dot{r}-\dot{r}_{f}\right)+\left(r-r_{d}\right) \\
= & {\left[\frac{2\left(a k_{1}-b k_{2}\right)}{I_{z}} \beta+\left(\frac{2\left(a^{2} k_{1}+b^{2} k_{2}\right)}{v_{x} I_{z}}+1\right) r\right.} \\
& \left.-\frac{2 a k_{1}}{I_{z}} \delta_{f}+\frac{2 b k_{2}}{I_{z}} \delta_{r}-r_{d}-\dot{r}_{d}\right]+\frac{\Delta M_{r}}{I_{z}}
\end{aligned}
$$

there is

$$
\dot{S}_{2}=\ddot{e}+\dot{e}=A+\frac{\Delta \dot{M}_{r}}{I_{z}}
$$

after derivation, where

$$
\begin{aligned}
A= & \frac{2\left(a k_{1}-b k_{2}\right)}{I_{z}}\left[\frac{2(a+b)}{m v_{x}} \beta+\left(\frac{2\left(a k_{1}-b k_{1}\right)}{m v_{x}^{2}}-1\right) r\right. \\
& \left.-\frac{2 a}{m v_{x}} \delta_{f}-\frac{2 b}{m v_{x}} \delta_{r}\right]+\left(\frac{2\left(a^{2} k_{1}+b^{2} k_{2}\right)}{v_{x} I_{z}}+1\right) \dot{r} \\
& -\frac{2 a k_{1}}{I_{z}} \dot{\delta}_{f}+\frac{2 b k_{2}}{I_{z}} \dot{\delta}_{r}-\dot{r}_{d}-\ddot{r}_{d} .
\end{aligned}
$$
then

Suppose $\dot{S}_{2}=-K_{r} \operatorname{sgn}(S)$,

$$
\Delta \dot{M}=-I_{z}\left[K_{r} \operatorname{sgn}\left(S_{2}\right)+A\right]
$$

where $k_{1}$ stands for roll stiffness of front suspension and $k_{2}$ stands for roll stiffness of rear suspension. Additional yaw moment $\Delta M_{r}$. needed by DYC can be obtained by integrating $\Delta \dot{M}_{r}$.

When $\mu$ is small, $\beta$ needs to be limited to ensure the stability of vehicle. Suppose $e=\beta-\beta_{d}$. The sliding mode surface is expressed as:

$$
\begin{aligned}
S_{3}= & \dot{e}+e=\left(\dot{\beta}-\dot{\beta}_{d}\right)+\left(\beta-\beta_{d}\right) \\
= & \left(\frac{2\left(k_{1}+k_{2}\right)}{m v_{x}}+1\right) \beta+\left(\frac{2\left(a k_{1}+b k_{2}\right)}{m v_{x}^{2}}-1\right) r \\
& -\frac{2 k_{1}}{m v_{x}} \delta_{f}-\frac{2 k_{2}}{m v_{x}} \delta_{r}-\dot{\beta}_{d}-\beta_{d}
\end{aligned}
$$

there is

$$
\dot{S}_{3}=\ddot{e}+\dot{e}=B+\left(\frac{2\left(a k_{1}+b k_{2}\right)}{m v_{x}^{2}}-1\right) \frac{\Delta M_{\beta}}{I_{z}}
$$

after derivation, where

$$
\begin{aligned}
B= & \left(\frac{2\left(k_{1}+k_{2}\right)}{m v_{x}}+1\right)\left[\frac{2\left(k_{1}+k_{2}\right)}{m v_{x}} \beta+\left(\frac{2\left(a k_{1}+b k_{2}\right)}{m v_{x}^{2}}-1\right) \beta\right. \\
& \left.-\frac{2 k_{1}}{m v_{x}} \delta_{f}-\frac{2 k_{2}}{m v_{x}} \delta_{r}\right]+\left(\frac{2\left(a k_{1}+b k_{2}\right)}{m v_{x}^{2}}-1\right) \\
\times & {\left[\frac{2\left(a k_{1}-b k_{2}\right)}{I_{z}} \beta+\left(\frac{2\left(a^{2} k_{1}+b^{2} k_{2}\right)}{v_{x} I_{z}}\right) r-\frac{2 a k_{1}}{I_{z}} \delta_{f}+\frac{2 b k_{2}}{I_{z}} \delta_{r}\right] } \\
& -\frac{2 k_{1}}{m v_{x}} \dot{\delta}_{f}-\frac{2 k_{2}}{m v_{x}} \dot{\delta}_{x}-\ddot{\beta}_{d}-\dot{\beta}_{d} .
\end{aligned}
$$

Suppose

$$
\dot{S}_{3}=-K_{\beta} \operatorname{sgn}\left(S_{3}\right),
$$

then

$$
\Delta M_{\beta}=I_{z}\left[B+K_{\beta} \operatorname{sgn}\left(S_{3}\right)\right]\left(1-\frac{2\left(a k_{1}+b k_{2}\right)}{m v_{x}^{2}}\right)^{-1} .
$$

In summary, the final output of the DYC controller is:

$$
\Delta M=K \Delta M_{r}+(1-K) \Delta M_{\beta} .
$$

When $|\beta|$ is relatively small, $r$ dominates; when $|\beta|$ is large, $\beta$ dominates. In this way, the influence of both $r$ and $\beta$ on the lateral stability of vehicle can be taken into account.

\subsection{Integration of ARS and DYC}

According to Hurwitz stability judgment, the vehicle stability conditions are:

$$
\begin{gathered}
c_{f} c_{r} l^{2}+m u^{2}\left(c_{r} l_{r}-c_{f} l_{f}\right)>0 \\
u_{c h}^{2}=\frac{c_{f} c_{r} l^{2}}{m\left(c_{r} l_{r}-c_{f} l_{f}\right)} \\
1+\frac{u^{2}}{u_{c h}^{2}}>0
\end{gathered}
$$

where $u$ represents the longitudinal speed and $u_{c h}$ stands for the characteristic speed. The method of determining the stability of vehicle is:

$$
\left\{\begin{array}{l}
1+\frac{u^{2}}{u_{c h}^{2}}<0, \text { unstable } \\
1+\frac{u^{2}}{u_{c h}^{2}}<0, \text { critical stable } \\
1+\frac{u^{2}}{u_{c h}^{2}}<0, \text { stable }
\end{array}\right.
$$




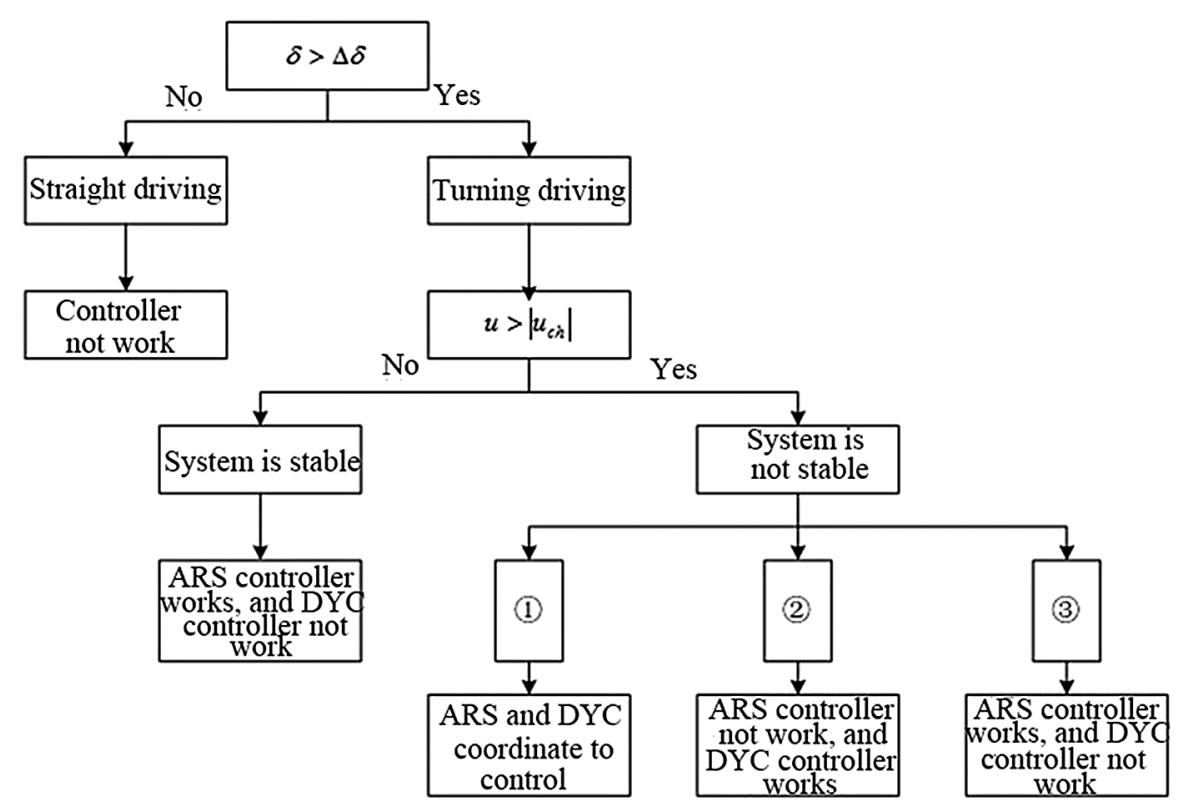

Fig. 1. Integrated control method.

Under the integrated control of ARS and DYC, the specific strategy is shown in Figure 1.

(1) $|r| \leq \frac{0.85 \mu g}{u}$ and ||$r|-| r_{d}||>\Delta \omega_{1}$ or $|r|>\frac{0.85 \mu g}{u}$ and $\Delta \omega_{1}<\| r|-| r_{d}|| \leq \Delta \omega_{2}$

(2) $|r|>\frac{0.85 \mu g}{u}$ and ||$r|-| r_{d}||>\Delta \omega_{2}$;

(3) other conditions except (1) and (2).

\section{Performance analysis of algorithm}

\subsection{Experimental parameters}

The integrated control method designed in this study was simulated under the environment of MATLAB/ SIMULINK. The simulated vehicle speed was set as $80 \mathrm{~km} / \mathrm{h}$. The vehicle parameters are shown in Table 1.

\subsection{Comparison of control performance}

When the ground adhesion coefficient was 0.2 , the vehicle control results are shown in Figures 2 and 3 and Table 2.

Based on Figures 2 and 3, it was found that the stability of the vehicle was poor under uncontrolled condition, values of $r$ and $\beta$ had large changes, indicating that the safety of the vehicle during driving was low; under the control of single DYC controller, the stability of the vehicle was improved to a certain extent; under the integrated control of ARS $+\mathrm{DYC}$, the stability of the vehicle was significantly improved, and changes of values of $r$ and $\beta$ are relatively stable. It was found from the comparison in Table 2 that the overshoot of the vehicle system under integrated control was smaller, the adjustment time was shorter, and the steady-state error was smaller. It showed that the control performance of the integrated control method was better and more stable than that of single
Table 1. Vehicle parameters.

\begin{tabular}{ll}
\hline Parameters & $\begin{array}{l}\text { Numerical } \\
\text { value }\end{array}$ \\
\hline Total weight of vehicle $m / \mathrm{kg}$ & 1231 \\
Front axle distance $l_{f} / \mathrm{m}$ & 1.18 \\
Rear axle distance $l_{r} / \mathrm{m}$ & 1.77 \\
Yam rotational inertia of vehicle $I_{z} / \mathrm{kg} \mathrm{m}^{2}$ & 1181.8 \\
Transmission ratio of steering wheel & 20 \\
to front wheel $n_{s w}$ & \\
Roll stiffness of front suspension $k_{1} /(\mathrm{Nm} / \mathrm{rad})$ & 47298 \\
Roll stiffness of rear suspension $k_{2} /(\mathrm{Nm} / \mathrm{rad})$ & 37311 \\
Distance from centroid to front axle $a / \mathrm{m}$ & 1.035 \\
Distance from centroid to rear axle $b / \mathrm{m}^{-1}$ & 1.655 \\
Cornering stiffness of front axle $c_{f} /\left(\mathrm{kN} \mathrm{rad}^{-1}\right)$ & 110 \\
Cornering stiffness of rear axle $c_{r} /\left(\mathrm{kN} \mathrm{rad}^{-1}\right)$ & 110 \\
\hline
\end{tabular}

control method, which is more conducive to the safe driving of vehicles.

\subsection{Control result under limit conditions}

The extreme conditions with cross-wind interference were simulated, and the applied crosswind action force is shown in Figure 4.

Under the influence of crosswind action force shown in Figure 4, the vehicle control results are shown in Figures 5 and 6.

It was found from Figures 5 and 6 that under the influence of crosswind action force, if the vehicle was not controlled, values of $r$ and $\beta$ had large changes, which seriously affected the safe driving of vehicles; under the 
Table 2. Control performance.

\begin{tabular}{lllll}
\hline & & Overshoot/\% & Adjustment time/s & Steady-state error \\
\hline Yaw velocity & DYC & 11 & 2.12 & -0.003 \\
& ARS + DYC & 2 & 1.33 & -0.001 \\
Centroid sideslip angle & DYC & 8 & 3.78 & -0.031 \\
& ARS + DYC & $/$ & 3.12 & -0.019 \\
\hline
\end{tabular}

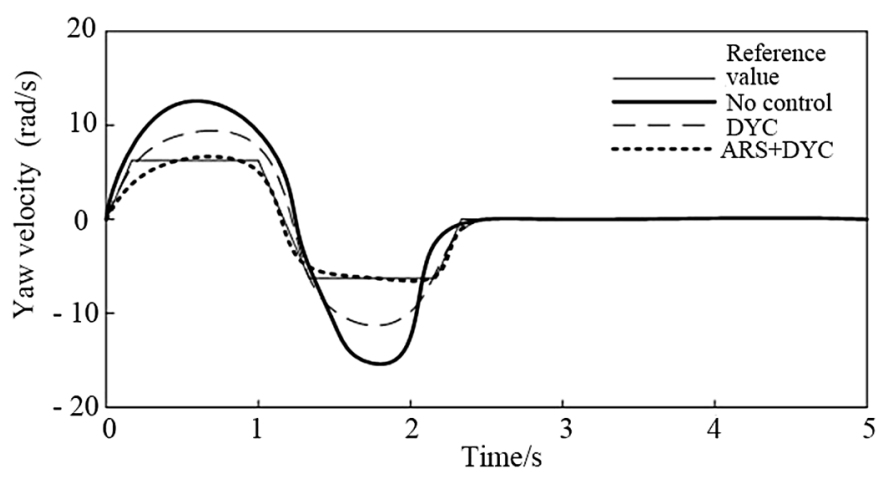

Fig. 2. Yaw velocity response.

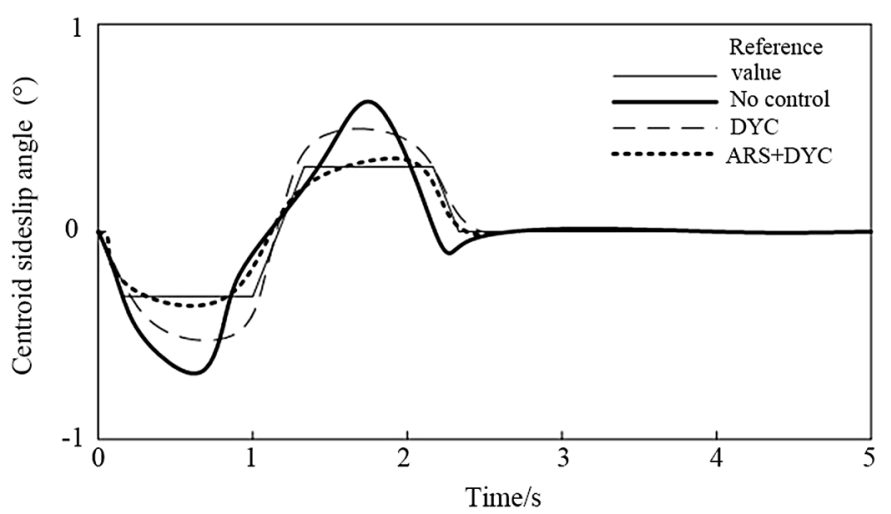

Fig. 3. Centroid sideslip angle response.

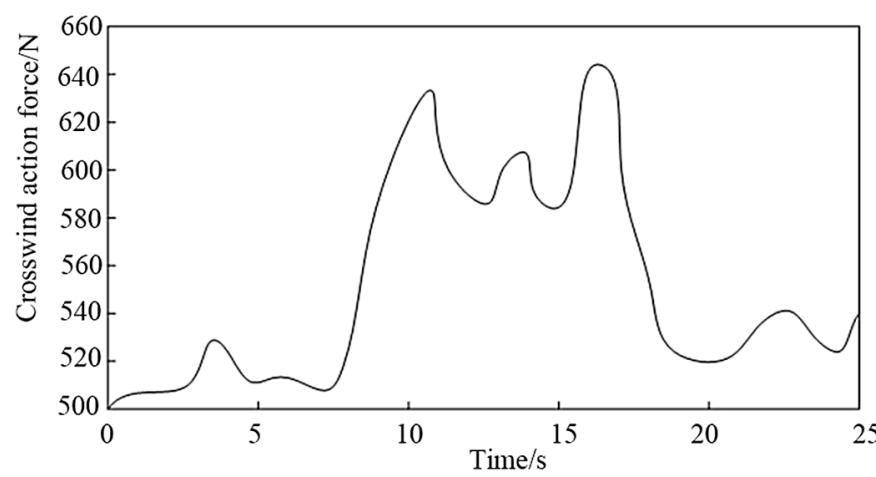

Fig. 4. Crosswind action force.

integrated control of $\mathrm{ARS}+\mathrm{DYC}$, values of $r$ and $\beta$ tracked the reference values well, and the errors between the control values and reference values were very small, which showed that the driving condition of the vehicle was

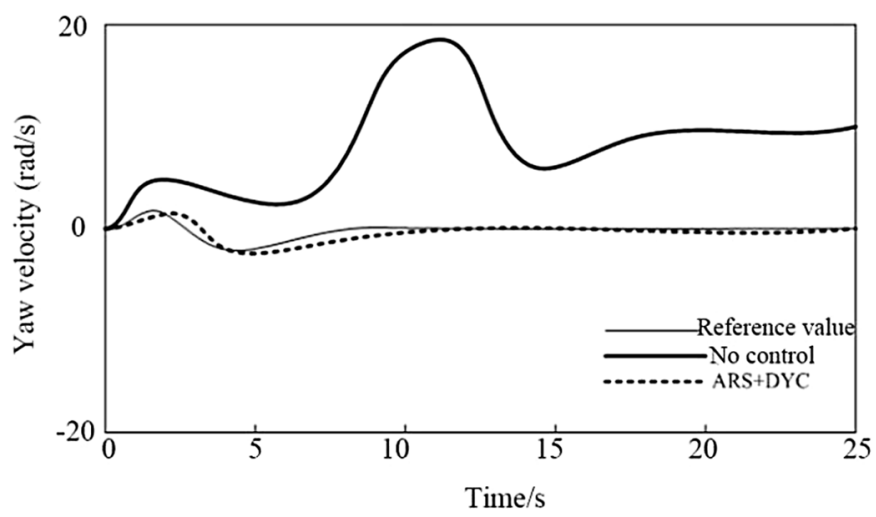

Fig. 5. Yaw velocity response.

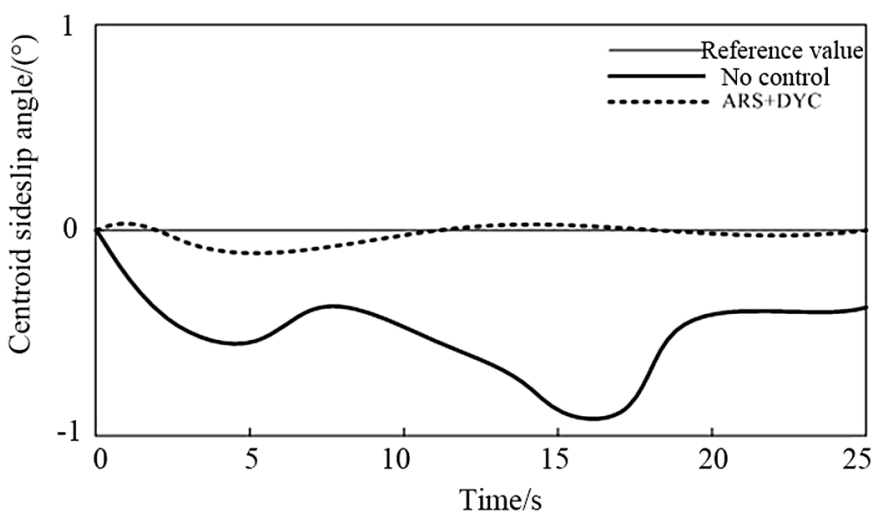

Fig. 6. Centroid side slip angle response.

relatively stable at this time. It was concluded that the integrated control had excellent performance under extreme conditions.

\section{Discussion}

With the development of economy, the use of vehicles has been more and more widespread, and the pollution is also more and more serious [11,12]. Moreover, as the main energy used by vehicles, the oil resource is decreasing and the oil price is rising, which makes vehicles have to seek other energy sources to replace oil. However, as far as the current situation is concerned, coal, natural gas and other resources still cannot be fully utilized because of some technical problems in cleaning and transportation. It is found that electric energy, as a secondary energy, can be well used as automotive energy. It has obvious advan- 
tages, such as less dependence on primary energy, easy acquisition and less pollution to the environment [13]. Compared with traditional vehicles, electric vehicles are environmentally friendly [14]. They can realize frontwheel steering, rear-wheel steering, four-wheel steering and other modes with high flexibility and controllable freedom. With the increasing complexity of electric vehicles, traditional independent control methods have not been able to meet the needs of vehicles, so integrated control appears. Integrated control can achieve coordinated work among different control systems and achieve better control effect. The integrated control method of ARS and DYC was studied in this study.

According to the experimental results, if the vehicle was not controlled, the fluctuations of values of $r$ and $\beta$ would be large during driving, which could lead to instability of the vehicle and severely threaten the safe driving of the vehicle. In the comparison between single DYC and ARS + DYC, the DYC controller could promote the stability of the vehicle, but it could not meet the need of safe driving; under the integrated control, the DYC controller could improve the stability of the vehicle, the variation of values of $r$ and $\beta$ was small, which was close to the reference value, indicating that the stability of the vehicle was good. The comparison of the performance between the two methods suggested that the integrated control method had smaller overshoot, higher regulation speed, and smaller steady-state error, i.e., having better performance than the single control method. The simulation results of extreme conditions suggested that the integrated control also showed good performance under the influence of crosswind, and values of $r$ and $\beta$ tracked the reference value well, achieving stable driving of the vehicle. According to the experimental results, it is found that the integrated control method of $\mathrm{ARS}+\mathrm{DYC}$ designed in this study plays a prominent role in achieving stable and safe driving of vehicles and it is worth further promotion and application in practice.

4WID vehicle is the only route for the future development of advanced vehicles as it can effectively solve problems of energy and environment. Study on its integrate $d$ control is of great values to improve the performance of vehicles. Therefore study on the integrated control of 4WID vehicles has broad prospect. Although some achievements have been made in the research of integrated control method, there are still many tasks to be further studied, such as:

- vehicle suspension control is not taken into account;

- the research is still at the level of simulation and needs to be tested in real vehicle;

- the control algorithms such as chattering suppression need to be further explored;

- the performance under other limited conditions of vehicles such as tyre pressure and ice snow covered pavement needs to be studied;

- more integrated control systems can be considered.

\section{Conclusion}

This paper presented a vehicle integrated control method with ARS and DYC working in coordination. The control methods of ARS and DYC were introduced, and the coordinated control strategy was designed. The simulation results showed that the integrated control had better performance than single control and could achieve stable and safe driving of vehicles. It also showed excellent performance under extreme conditions. This work makes some contributions to the improvement of the control performance of electric vehicles and the further application of electric vehicles in the life.

\section{References}

1. L.H. Liu, Key Eng. Mater. 93, 8 (2016)

2. Q. Meng et al., Int. J. Syst. Sci. 49, 1518-1528 (2018)

3. Z.L. Liao et al., Acta. Armamentarii 38, 833-842 (2017)

4. J. Wu et al., Sci. China Technol. Sci. 58, 75-85 (2015)

5. J. Zhao et al., Vehicle. Syst. Dyn. 55, 72-103 (2017)

6. H. Shen, Y.S. Tan, Mod. Phys. Lett. B 31, 19-21 (2017)

7. S. Ding, J. Sun, Nonlinear. Dynam. 88, 1-16 (2016)

8. H. Shen et al., J. Comput. Theor. Nanos. 13, 2043-2048 (2016)

9. H.Y. Ma, C.P. Li, Z.F. Wang, Energy Proc. 105, 2310-2316 (2017)

10. T. Kobayashi et al., Vehicle Syst. Dyn. 56, 719-733 (2018)

11. K.M. Tan, V.K. Ramachandaramurthy, J.Y. Yong, Renew. Sust. Energ. Rev. 53, 720-732 (2016)

12. M.A. Hannan et al., Renew. Sust. Energ. Rev. 78, 834-854(2017)

13. N. Andrenacci, R. Ragona, G. Valenti, Appl. Energ. 182, 39-46 (2016)

14. Y. Cao et al., IEEE Commun. Mag. 56, 150-156 (2018)

Cite this article as: Ling Yu, Sunan Yuan, Integrated control performance of drive-by-wire independent drive electric vehicle, Int. J. Metrol. Qual. Eng. 10, 16 (2019) 